\title{
A Fully Abstract Symbolic Semantics for Psi-Calculi
}

\author{
Magnus Johansson \\ Björn Victor \\ Joachim Parrow
}

\begin{abstract}
We present a symbolic transition system and bisimulation equivalence for psi-calculi, and show that it is fully abstract with respect to bisimulation congruence in the non-symbolic semantics.

A psi-calculus is an extension of the pi-calculus with nominal data types for data structures and for logical assertions representing facts about data. These can be transmitted between processes and their names can be statically scoped using the standard pi-calculus mechanism to allow for scope migrations. Psi-calculi can be more general than other proposed extensions of the pi-calculus such as the applied pi-calculus, the spi-calculus, the fusion calculus, or the concurrent constraint pi-calculus.

Symbolic semantics are necessary for an efficient implementation of the calculus in automated tools exploring state spaces, and the full abstraction property means the semantics of a process does not change from the original.
\end{abstract}

\section{Introduction}

A multitude of extensions of the pi-calculus have been defined, allowing higher-level data structures and operations on them to be used as primitives when modelling applications. Ranging from integers, lists, or booleans to encryption/decryption or hash functions, the extensions increase the applicability of the basic calculus. In order to implement automated tools for analysis and verification using state space exploration (e.g. bisimilarity or model checking), each extended calculus needs a symbolic semantics, where the state space of agents is reduced to a manageable size - the non-symbolic semantics typically generates infinite state spaces even for very simple agents.

The extensions thus require added efforts both in developing the theory of the calculus for each variant, and in constructing specialised symbolic semantics for them. As the complexity of the extensions increases, producing correct results in these areas can be very hard. For example the labelled semantics of applied pi-calculus [2] and of CC-Pi [14] have both turned out to be non-compositional; another example is the rather complex bisimulations which have been developed for the spi-calculus [3] (see [12] for an overview of non-symbolic bisimulations, or [11, 13, 10] for symbolic ones).

The psi-calculi [5] improve the situation: a single framework allows a range of specialised calculi to be formulated with a lean and compositional labelled semantics: with the parameters appropriately instantiated, the resulting calculus can be used to model applications such as cryptographic protocols and concurrent constraints, but also more advanced scenarios with polyadic synchronization or higher-order data and logics. The expressiveness and modelling convenience of psi-calculi exceeds that of earlier pi-calculus extensions, while the purity of the semantics is on par with the original pi-calculus. Its metatheory has been proved mechanically using the theorem prover Isabelle [6].

In this paper we develop a symbolic semantics for psi-calculi, admitting large parts of this range of calculi to be verified more efficiently. We define a symbolic version of labelled bisimulation equivalence, and show that it is fully abstract with respect to bisimulation congruence in the original semantics. This means that our new symbolic semantics does not change which processes are considered equivalent.

A symbolic semantics abstracts the values received in an input action. Instead of a possibly infinite branching of concrete values, a single name is used to represent them all. When the received values are used in conditional constructions (e.g. if-then-else) or as communication channels, we do not know their

B. Klin, P. Sobociński (Eds.):

6th Workshop on Structural Operational Semantics (SOS'09)

EPTCS 18, 2010, pp. 17-31 doi 10.4204/EPTCS.18.2 
precise value, but need to record the constraints which must be satisfied for a resulting transition to be valid.

A (non-symbolic) psi-calculus transition has the form $\Psi \triangleright P \stackrel{\alpha}{\longrightarrow} P^{\prime}$, with the intuition that $P$ can perform $\alpha$ leading to $P^{\prime}$ in an environment that asserts $\Psi$. For example, if $P$ can do an $\alpha$ to $P^{\prime}$ then case prime $(x): P$ can make an $\alpha$-transition to $P^{\prime}$ if we can deduce prime $(x)$ from the environment, e.g.

$$
\{x=3\} \triangleright \text { case } \operatorname{prime}(x): P \stackrel{\alpha}{\longrightarrow} P^{\prime} .
$$

In the symbolic semantics where we may not have the precise value of $x$, we instead decorate the transition with its requirement, so

$$
\Psi \triangleright \text { case prime }(x): P \underset{C \wedge\{\Psi \vdash \operatorname{prime}(x)\}}{\stackrel{\alpha}{\longrightarrow}} P^{\prime} \text { (for any } \Psi \text { ) }
$$

where $C$ is the requirement for $P$ to do an $\alpha$ to $P^{\prime}$ in the environment $\Psi$. Constraints also arise from communication between parallel agents, where, in the symbolic case, the precise channels may not be known; instead we allow communication over symbolic representations of channels and record the requirement in a transition constraint. As an example consider $a(x) \cdot a(y) \cdot(\bar{x} x \cdot P \mid y(z) \cdot Q)$ which after its initial inputs only has symbolic values of $x$ and $y$. The resulting agent has the symbolic transition $\bar{x} x . P|y(z) \cdot Q \underset{\{\Psi \vdash x \dot{\leftrightarrow} y\}}{\stackrel{\tau}{\longrightarrow}} P| Q[z:=x]$ where $x \dot{\leftrightarrow} y$ means that $x$ and $y$ represent the same channel, but might not have a $\tau$ transition in the non-symbolic semantics.

Communication channels in psi-calculi may be structured data terms, not only names. This leads to a new source of possibly infinite branching: a subject in a prefix may be rewritten to another equivalent term before it is used in a transition. E.g., when first $(x, y)$ and $x$ represent the same channel, $P=\overline{\text { first }(a, b)} c \cdot P^{\prime} \stackrel{\bar{a} c}{\longrightarrow} P^{\prime}$, but also $P \stackrel{\overline{\text { first }(a, c) c}}{\longrightarrow} P^{\prime}$, etc. The possibility of using structured channels gives significant expressive power (see [5]). Our symbolic semantics abstracts the equivalent forms of channel subject by using a fresh name as subject, and adds a suitable constraint to the transition label (see Section 3).

\subsection{Comparison to related work}

Symbolic bisimulations for process calculi have a long history. Our work is to a large extent based on the pioneering work by Hennessy and Lin [17] for value-passing CCS, later specialised for the pi-calculus by Boreale and De Nicola [9] and independently by Lin [18, 19]. While [17] is parameterised by general boolean expressions on an underlying data signature it does not handle names and mobility; on the other hand [9, 18, 19] handle only names and no other data structures. The number of (direct or indirect) follow-up works to these is huge, with applications ranging from pi-calculus to constraint programming; here we focus on the relation to the ones for applied pi-calculus and spi-calculus.

The existing tools for calculi based on the applied pi-calculus (e.g. [1, 7, 8]), are not fully abstract wrt bisimulation. A symbolic semantics and bisimulation for applied pi-calculus has been defined in [15], but it is not complete. Additionally, the labelled (non-symbolic) bisimulation of applied pi-calculus is not compositional (see [5]). The situation for the spi-calculus is better: fully abstract symbolic bisimulation for hedged bisimulation has been defined in [10], and for open hedged bisimulation (a finer equivalence) in [13]. According to those authors, neither is directly mechanizable. The only symbolic bisimulation which to our knowledge has been implemented in a tool is not fully abstract [11].

It can be argued [11] that incompleteness is not a problem when verifying authentication and secrecy properties of security protocols, which appears to have been the main application of the applied picalculus so far. When going beyond security analysis we claim (based on experience from the Mobility 
Workbench [22]) that completeness is very important: when analysing agents with huge state spaces, a positive result (the agents are equivalent) may be more difficult to achieve than a negative result (the agents differ). However, such a negative result can only be trusted if the analysis is fully abstract.

Our symbolic semantics is relatively simple, compared to the ones presented for applied pi-calculus or spi-calculus. In relation to the former, we are helped significantly by the absence of structural equivalence rules, which in applied pi-calculus are rather complex. In [15] an intermediate semantics is used to overcome the complexity. In contrast we can directly relate the original and symbolic semantics. In relation to the symbolic semantics for spi-calculus, our semantics has a straight-forward treatment of scope opening due to the simpler psi-calculi semantics. In addition, the complexities of spi-calculus bisimulations are necessarily inherited by the symbolic semantics, introducing e.g. explicit environment knowledge representations with timestamps on messages and variables. In psi-calculi, bisimulation is much simpler and the symbolic counterpart is not significantly more complex than the one for valuepassing CCS.

In the light of these complications, the relevance of precise encodings of the applied pi-calculus or spi-calculus as psi-calculi, or comparing the resulting bisimulation equivalences is questionable. Our interest is in handling and analysing the same type of applications, and also the more advanced kinds of applications mentioned in the beginning of this section.

Disposition. In the next section we review the basic definitions of syntax, semantics, and bisimulation of psi-calculi. Section 3 presents the symbolic semantics and bisimulation, while Section 4 illustrates the concrete and symbolic transitions and bisimulations by examples. In Section 5 we show our main results: the correspondence between concrete and symbolic transitions and bisimulations. Section 6 concludes, and presents plans and ideas for future work.

\section{Psi-calculi}

This section is a brief recapitulation of psi-calculi and nominal data types; for a more extensive treatment including motivations and examples see [5].

\subsection{Nominal data types}

We assume a countably infinite set of atomic names $\mathscr{N}$ ranged over by $a, b, \ldots, x, y, z$. Intuitively, names will represent the symbols that can be statically scoped, and also represent symbols acting as variables in the sense that they can be subjected to substitution. A nominal set [20, 16] is a set equipped with name swapping functions written $(a b)$, for any names $a, b$. An intuition is that for any member $X$ it holds that $(a b) \cdot X$ is $X$ with $a$ replaced by $b$ and $b$ replaced by $a$. One main point of this is that even though we have not defined any particular syntax we can define what it means for a name to "occur" in an element: it is simply that it can be affected by swappings. The names occurring in this way in an element $X$ constitute the support of $X$, written $\mathrm{n}(X)$.

We write $a \# X$, pronounced " $a$ is fresh for $X$ ", for $a \notin \mathrm{n}(X)$. If $A$ is a set of names we write $A \# X$ to mean $\forall a \in A$. $a \# X$. We require all elements to have finite support, i.e., $\mathrm{n}(X)$ is finite for all $X$.

A function $f$ on nominal sets is equivariant if $(a b) \cdot f(X)=f((a b) \cdot X)$ holds for all $X, a, b$, and similarly for functions and relations of any arity. Intuitively, this means that all names are treated equally.

A nominal data type is just a nominal set together with a set of functions on it. In particular we require a substitution function [6], which intuitively substitutes elements for names. If $X$ is an element of 
a data type, $\tilde{a}$ is a sequence of names without duplicates and $\tilde{Y}$ is an equally long sequence of elements, the substitution $X[\tilde{a}:=\tilde{Y}]$ is an element of the same data type as $X$.

\subsection{Agents}

A psi-calculus is defined by instantiating three nominal data types and four operators:

Definition 1 (Psi-calculus parameters). A psi-calculus requires the three (not necessarily disjoint) nominal data types:

$$
\begin{aligned}
& \text { T the (data) terms, ranged over by } M, N \\
& \text { C the conditions, ranged over by } \varphi \\
& \text { A the assertions, ranged over by } \Psi
\end{aligned}
$$

and the four equivariant operators:

$$
\begin{array}{ll}
\dot{\leftrightarrow}: \mathbf{T} \times \mathbf{T} \rightarrow \mathbf{C} & \text { Channel Equivalence } \\
\otimes: \mathbf{A} \times \mathbf{A} \rightarrow \mathbf{A} & \text { Composition } \\
\mathbf{1}: \mathbf{A} & \text { Unit } \\
\vdash \subseteq \mathbf{A} \times \mathbf{C} & \text { Entailment }
\end{array}
$$

The binary functions above will be written in infix. Thus, if $M$ and $N$ are terms then $M \dot{\leftrightarrow} N$ is a condition, pronounced " $M$ and $N$ are channel equivalent" and if $\Psi$ and $\Psi^{\prime}$ are assertions then so is $\Psi \otimes \Psi^{\prime}$. Also we write $\Psi \vdash \varphi$, “ $\Psi$ entails $\varphi$ ”, for $(\Psi, \varphi) \in \vdash$.

The data terms are used to represent all kinds of data, including communication channels. Conditions are used as guards in agents, and $M \dot{\leftrightarrow} N$ is a particular condition saying that $M$ and $N$ represent the same channel. The assertions will be used to declare information necessary to resolve the conditions. Assertions can be contained in agents and thus represent information postulated by that agent; they can contain names and thereby be syntactically scoped and thus represent information known only to the agents within that scope. The intuition of entailment is that $\Psi \vdash \varphi$ means that given the information in $\Psi$, it is possible to infer $\varphi$. We say that two assertions are equivalent if they entail the same conditions:

Definition 2 (Assertion equivalence). Two assertions are equivalent, written $\Psi \simeq \Psi^{\prime}$, iffor all $\varphi$ we have that $\Psi \vdash \varphi \Leftrightarrow \Psi^{\prime} \vdash \varphi$.

A psi-calculus is formed by instantiating the nominal data types and morphisms so that the following requisites are satisfied:

Definition 3 (Requisites on valid psi-calculus parameters).

$$
\begin{aligned}
& \text { Channel Symmetry: } \quad \Psi \vdash M \dot{\leftrightarrow} N \Longrightarrow \Psi \vdash N \dot{\leftrightarrow} M \\
& \text { Channel Transitivity: } \quad \Psi \vdash M \dot{\leftrightarrow} N \wedge \Psi \vdash N \leftrightarrow L \\
& \Longrightarrow \Psi \vdash M \dot{\leftrightarrow} L \\
& \text { Weakening: } \quad \Psi \vdash \varphi \Longrightarrow \Psi \otimes \Psi^{\prime} \vdash \varphi \\
& \text { Composition: } \quad \Psi \simeq \Psi^{\prime} \Longrightarrow \Psi \otimes \Psi^{\prime \prime} \simeq \Psi^{\prime} \otimes \Psi^{\prime \prime} \\
& \text { Identity: } \quad \Psi \otimes \mathbf{1} \simeq \Psi \\
& \text { Associativity: } \quad\left(\Psi \otimes \Psi^{\prime}\right) \otimes \Psi^{\prime \prime} \simeq \Psi \otimes\left(\Psi^{\prime} \otimes \Psi^{\prime \prime}\right) \\
& \text { Commutativity: } \quad \Psi \otimes \Psi^{\prime} \simeq \Psi^{\prime} \otimes \Psi
\end{aligned}
$$


Our requisites on a psi-calculus are that the channel equivalence is a partial equivalence relation, that $\otimes$ preserves equivalence, and that the equivalence classes of assertions form an abelian monoid. We do not require that channel equivalence is reflexive. There may be terms $M$ such that $M \dot{\leftrightarrow} M$ does not hold. By transitivity and symmetry then $M \dot{\leftrightarrow} N$ holds for no $N$, which means that $M$ cannot be used as a channel at all. In this way we accommodate data structures which cannot be used as channels. The requisite of weakening (which is not present in [5]) excludes some non-monotonic logics; it simplifies our proofs in the present paper although we do not know if it is absolutely necessary. It is only used in one place in the proof of Theorem 20 .

In the following $\tilde{a}$ means a finite (possibly empty) sequence of names, $a_{1}, \ldots, a_{n}$. The empty sequence is written $\varepsilon$ and the concatenation of $\tilde{a}$ and $\tilde{b}$ is written $\tilde{a} \tilde{b}$. When occurring as an operand of a set operator, $\tilde{a}$ means the corresponding set of names $\left\{a_{1}, \ldots, a_{n}\right\}$. We also use sequences of terms, conditions, assertions etc. in the same way.

A frame can intuitively be thought of as an assertion with local names:

Definition 4 (Frame). A frame $F$ is of the form $(v \widetilde{b}) \Psi$ where $\widetilde{b}$ is a sequence of names considered bound in the assertion $\Psi$. We use $F, G$ to range over frames. ${ }^{1}$

Name swapping on a frame $F=(v \widetilde{b}) \Psi$ just distributes to its two components. We identify alpha equivalent frames, so $\mathrm{n}(F)=\mathrm{n}(\Psi)-\mathrm{n}(\widetilde{b})$. We overload 1 to also mean the least informative frame $(v \varepsilon) \mathbf{1}$ and $\otimes$ to mean composition on frames defined by $\left(v \widetilde{b}_{1}\right) \Psi_{1} \otimes\left(v \widetilde{b}_{2}\right) \Psi_{2}=\left(v \widetilde{b}_{1} \widetilde{b}_{2}\right) \Psi_{1} \otimes \Psi_{2}$ where $\widetilde{b}_{1} \# \widetilde{b}_{2}, \Psi_{2}$ and vice versa. We write $(v c)((v \widetilde{b}) \Psi)$ for $(v c \widetilde{b}) \Psi$, and when there is no risk of confusing a frame with an assertion we write $\Psi$ for $(v \varepsilon) \Psi$.

Definition 5 (Equivalence of frames). We define $F \vdash \varphi$ to mean that there exists an alpha variant $(v \widetilde{b}) \Psi$ of $F$ such that $\widetilde{b} \# \varphi$ and $\Psi \vdash \varphi$. We also define $F \simeq G$ to mean that for all $\varphi$ it holds that $F \vdash \varphi$ iff $G \vdash \varphi$.

Intuitively a condition is entailed by a frame if it is entailed by the assertion and does not contain any names bound by the frame. Two frames are equivalent if they entail the same conditions.

Definition 6 (Psi-calculus agents). Given valid psi-calculus parameters as in Definitions 1 and 3 the psi-calculus agents, ranged over by $P, Q, \ldots$, are of the following forms.

$\begin{array}{ll}\bar{M} N . P & \text { Output } \\ \underline{M}(x) . P & \text { Input } \\ \text { case } \varphi_{1}: P_{1} \square \cdots \square \varphi_{n}: P_{n} & \text { Case } \\ (v a) P & \text { Restriction } \\ P \mid Q & \text { Parallel } \\ ! P & \text { Replication } \\ (\Psi) & \text { Assertion }\end{array}$

In the Input $\underline{M}(x) . P, x$ binds its occurrences in P. Restriction binds a in P. An assertion is guarded if it is a subterm of an Input or Output. In a replication !P there may be no unguarded assertions in $P$.

In the Output and Input forms $M$ is called the subject and $N$ and $x$ the objects, respectively. Output and Input are similar to those in the pi-calculus, but arbitrary terms can function as both subjects and objects. Note that differently from [5], for simplicity the input is not pattern matching (see Section 6 for

\footnotetext{
${ }^{1}$ In some presentations frames have been written just as pairs $\langle\widetilde{b}, \Psi\rangle$. The notation in this paper better conveys the idea that the names bind into the assertion, at the slight risk of confusing frames with agents. Formally, we establish frames and agents as separate types, although a valid intuition is to regard a frame as a special kind of agent, containg only scoping and assertions. This is the view taken in [2].
} 
a discussion). The case construct works by performing the action of any $P_{i}$ for which the corresponding $\varphi_{i}$ is true. So it embodies both an if (if there is only one branch) and an internal nondeterministic choice (if the conditions are overlapping).

Some notational conventions: We define the agent $\mathbf{0}$ as $(\mathbf{1})$. The construct case $\left.\left.\varphi_{1}: P_{1}\right] \cdots\right] \varphi_{n}: P_{n}$ is sometimes written as case $\widetilde{\varphi}: \widetilde{P}$, or if $n=1$ as if $\varphi_{1}$ then $P_{1}$. The input subject is underlined to facilitate parsing of complicated expressions; in simple cases we often conform to a more traditional notation and omit the underline.

Formally, we define name swapping on agents by distributing it over all constructors, and substitution on agents by distributing it and avoiding captures by binders through alpha-conversion in the usual way. We identify alpha-equivalent agents; in that way we get a nominal data type of agents where the support $\mathrm{n}(P)$ of $P$ is the union of the supports of the components of $P$, removing the names bound by Input and $v$, and corresponds to the names with a free occurrence in $P$.

Definition 7 (Frame of an agent). The frame $\mathscr{F}(P)$ of an agent $P$ is defined inductively as follows:

$$
\begin{aligned}
& \mathscr{F}(\underline{M}(x) \cdot P)=\mathscr{F}(\bar{M} N \cdot P)= \\
& \mathscr{F}(\text { case } \widetilde{\varphi}: \widetilde{P})=\mathscr{F}(! P)=\mathbf{1} \\
& \mathscr{F}((\Psi))=(v \varepsilon) \Psi \\
& \mathscr{F}(P \mid Q)=\mathscr{F}(P) \otimes \mathscr{F}(Q) \\
& \mathscr{F}((v b) P)=(v b) \mathscr{F}(P)
\end{aligned}
$$

\subsection{Operational semantics}

The presentation of psi-calculi in [5] gives a semantics of an early kind, where input actions are of kind $\underline{M} N$. Here we give an operational semantics of the late kind, meaning that the labels of input transitions contain variables for the object to be received. With this kind of semantics it is easier to establish a relation to the symbolic semantics. We also establish precisely how it relates to the original.

Definition 8 (Actions). The actions ranged over by $\alpha, \beta$ are of the following three kinds: $\bar{M}(v \tilde{a}) N$ (Output), $\underline{M}(x)$ (Input), and $\tau$ (Silent).

For actions we refer to $M$ as the subject and $N$ and $x$ as the objects. We let $\operatorname{subj}(\bar{M}(v \widetilde{a}) N)=$ $\operatorname{subj}(\underline{M}(x))=M$. We define $\operatorname{bn}(\bar{M}(v \tilde{a}) N)=\tilde{a}, \operatorname{bn}(\underline{M}(x))=\{x\}$, and $\operatorname{bn}(\tau)=\emptyset$. We also define $\mathrm{n}(\tau)=\emptyset$ and $\mathrm{n}(\alpha)=\mathrm{n}(N) \cup \mathrm{n}(M)$ if $\alpha$ is an output or input. As in the pi-calculus, the output $\bar{M}(v \tilde{a}) N$ represents an action sending $N$ along $M$ and opening the scopes of the names $\tilde{a}$. Note in particular that the support of this action includes $\tilde{a}$. Thus $\bar{M}(v a) a$ and $\bar{M}(v b) b$ are different actions.

Definition 9 (Transitions). A transition is of the kind $\Psi \triangleright P \stackrel{\alpha}{\longrightarrow} P^{\prime}$, meaning that when the environment contains the assertion $\Psi$ the agent $P$ can do an $\alpha$ to become $P^{\prime}$. The transitions are defined inductively in Table 1

Note that $\Psi$ in Table 1 expresses the effect that the environment has on the agent, by enabling conditions in CASE, by giving rise to action subjects in IN and OUT and by enabling interactions in COM.

Both agents and frames are identified by alpha equivalence. This means that we can choose the bound names fresh in the premise of a rule. In a transition the names in $b n(\alpha)$ count as binding into both the action object and the derivative, and transitions are identified up to alpha equivalence. This means that the bound names can be chosen fresh, substituting each occurrence in both the object and the derivative. This is the reason why bn $(\alpha)$ is in the support of the output action: otherwise it could be alpha-converted in the action alone.

Table 2 gives the rules for input and communication of an early kind used in [5]. The following lemma clarifies the relation between the two semantics: 


$$
\begin{aligned}
& \text { IN } \frac{\Psi \vdash M \dot{\leftrightarrow} K}{\Psi \triangleright \underline{M}(x) . P \stackrel{\underline{K}(x)}{\longrightarrow} P} \text { OUT } \frac{\Psi \vdash M \dot{\leftrightarrow} K}{\Psi \triangleright \bar{M} N . P \stackrel{\bar{K} N}{\longrightarrow} P} \text { CASE } \frac{\Psi \triangleright P_{i} \stackrel{\alpha}{\longrightarrow} P^{\prime} \quad \Psi \vdash \varphi_{i}}{\Psi \triangleright \text { case } \widetilde{\varphi}: \widetilde{P} \stackrel{\alpha}{\longrightarrow} P^{\prime}} \\
& \operatorname{CoM} \frac{\Psi_{Q} \otimes \Psi \triangleright P \stackrel{\bar{M}(v \widetilde{a}) N}{\longrightarrow} P^{\prime} \quad \Psi_{P} \otimes \Psi \triangleright Q \stackrel{\underline{K}(x)}{\longrightarrow} Q^{\prime} \quad \Psi \otimes \Psi_{P} \otimes \Psi_{Q} \vdash M \dot{\leftrightarrow} K}{\Psi \triangleright P \mid Q \stackrel{\tau}{\longrightarrow}(v \widetilde{a})\left(P^{\prime} \mid Q^{\prime}[x:=N]\right)} \widetilde{a} Q \\
& \operatorname{PAR} \frac{\Psi_{Q} \otimes \Psi \triangleright P \stackrel{\alpha}{\longrightarrow} P^{\prime}}{\Psi \triangleright P\left|Q \stackrel{\alpha}{\longrightarrow} P^{\prime}\right| Q} \operatorname{bn}(\alpha) \# Q \quad \text { SCOPE } \frac{\Psi \triangleright P \stackrel{\alpha}{\longrightarrow} P^{\prime}}{\Psi \triangleright(v b) P \stackrel{\alpha}{\longrightarrow}(v b) P^{\prime}} b \# \alpha, \Psi
\end{aligned}
$$

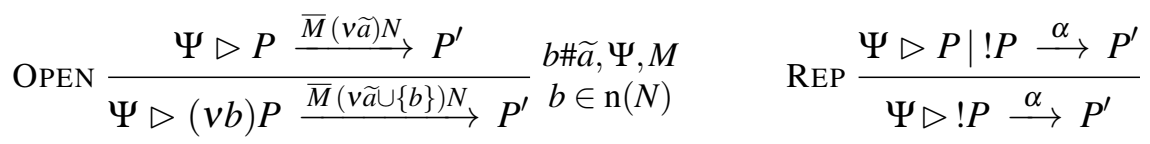

Table 1: Late operational semantics. Symmetric versions of COM and PAR are elided. In the rule COM we assume that $\mathscr{F}(P)=\left(v \widetilde{b}_{P}\right) \Psi_{P}$ and $\mathscr{F}(Q)=\left(v \widetilde{b}_{Q}\right) \Psi_{Q}$ where $\widetilde{b}_{P}$ is fresh for all of $\Psi, \widetilde{b}_{Q}, Q, M$ and $P$, and that $\widetilde{b}_{Q}$ is correspondingly fresh. In the rule PAR we assume that $\mathscr{F}(Q)=\left(v \widetilde{b}_{Q}\right) \Psi_{Q}$ where $\widetilde{b}_{Q}$ is fresh for $\Psi, P$ and $\alpha$. In OPEN the expression $\tilde{a} \cup\{b\}$ means the sequence $\tilde{a}$ with $b$ inserted anywhere.

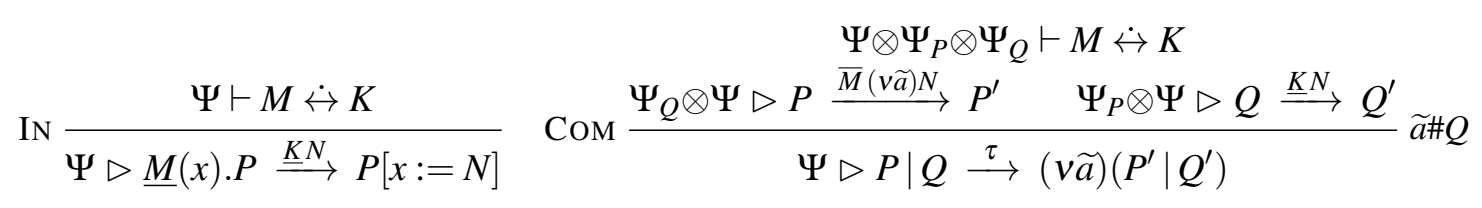

Table 2: Early structured operational semantics. All other rules are as in the late semantics of Fig. 1.

\section{Lemma 10.}

1. $\Psi \triangleright P \stackrel{M N}{\longrightarrow} Q$ in the early semantics iff there exist $Q^{\prime}$ and $x$ such that $\Psi \triangleright P \stackrel{M(x)}{\longrightarrow} Q^{\prime}$ in the late semantics, where $Q=Q^{\prime}[x:=N]$.

2. For output and $\tau$ actions, $\Psi \triangleright P \stackrel{\alpha}{\longrightarrow} Q$ in the early semantics iff the same transition can be derived in the early semantics.

The proof is by induction over the transition derivations. In the proof of (2), the case $\alpha=\tau$ needs both (1) and the case where $\alpha$ is an output.

\subsection{Bisimulation}

We proceed to define early bisimulation with the late semantics:

Definition 11 ((Early) Bisimulation). $A$ bisimulation $\mathscr{R}$ is a ternary relation between assertions and pairs of agents such that $\mathscr{R}(\Psi, P, Q)$ implies all of

1. Static equivalence: $\Psi \otimes \mathscr{F}(P) \simeq \Psi \otimes \mathscr{F}(Q)$ 
2. Symmetry: $\mathscr{R}(\Psi, Q, P)$

3. Extension of arbitrary assertion: $\forall \Psi^{\prime} . \mathscr{R}\left(\Psi \otimes \Psi^{\prime}, P, Q\right)$

4. Simulation: for all $\alpha, P^{\prime}$ such that $\operatorname{bn}(\alpha) \# \Psi, Q$

(a) if $\alpha=\underline{M}(x): \quad \Psi \triangleright P \stackrel{\alpha}{\longrightarrow} P^{\prime} \Longrightarrow$

$\forall L \exists Q^{\prime} . \Psi \triangleright Q \stackrel{\alpha}{\longrightarrow} Q^{\prime}$ and $\mathscr{R}\left(\Psi, P^{\prime}[x:=L], Q^{\prime}[x:=L]\right)$.

(b) otherwise: $\quad \Psi \triangleright P \stackrel{\alpha}{\longrightarrow} P^{\prime} \Longrightarrow \exists Q^{\prime} . \Psi \triangleright Q \stackrel{\alpha}{\longrightarrow} Q^{\prime}$ and $\mathscr{R}\left(\Psi, P^{\prime}, Q^{\prime}\right)$.

We define $P \dot{\sim} Q$ to mean that there exists a bisimulation $\mathscr{R}$ such that $\mathscr{R}(\mathbf{1}, P, Q)$. We also define $P \sim Q$ to mean that $P[x:=L] \dot{\sim} Q[x:=L]$ for all $x, L$.

The relation between this definition and the original definition of bisimulation in [5] is clarified by the following:

Lemma 12. For the psi-calculi in the present paper, a relation is a bisimulation according to Def. 11 precisely if it is a bisimulation according to [5].

The proof is straightforward using Lemma 10 . As a corollary the algebraic properties of $\sim$ established in [5] hold, notably that it is a congruence.

\section{Symbolic semantics and equivalence}

The idea behind a symbolic semantics is to reduce the state space of agents. One standard way is to avoid infinite branching in inputs by using a fresh name to represent whatever was received.

In psi-calculi there is an additional source of infinite branching: a subject in a prefix may get rewritten to many terms. Also here we use a fresh name to represent these terms. This means that the symbolic actions are the same as the concrete actions with the exception that only names are used as subjects.

A symbolic transition is of form

$$
\Psi \triangleright P \underset{C}{\stackrel{\alpha}{\rightarrow}} P^{\prime}
$$

The intuition is that this represents a set of concrete transitions, namely those that satisfy the constraint $C$. Before the formal definitions we here briefly explain the rationale. Consider a psi-calculus with integers and integer equations; for example a condition can be " $x=3$ ". An example agent is $P=$ case $x=3: P^{\prime}$. If $P^{\prime} \underset{\text { true }}{\stackrel{\alpha}{\longrightarrow}} P^{\prime \prime}$, where true is a constraint that is always true, then there should clearly be a transition $P \underset{C}{\stackrel{\alpha}{\rightarrow}} P^{\prime \prime}$ for some constraint $C$ that captures that $x$ must be 3 . One context that can make this constraint true is an input, as in $a(x) . P$. The input will give rise to a substitution for $x$, and if the substitution sends $x$ to 3 the constraint is satisfied. In this way the constraints are similar to those for the pi-calculus [9, 18]. In psi-calculi there is an additional way that a context can enable the transition: it can contain an assertion as in $(x:=3) \mid P$. Concretely this agent has a transition $(x:=3)|P \stackrel{\alpha}{\longrightarrow}(x:=3)| P^{\prime \prime}$ since $x:=3 \vdash x=3$. Therefore a solution of a constraint will contain both a substitution of terms for names (representing the effect of an input) and an assertion (representing the effect of a parallel component).

Definition 13. The atomic constraints are of the form $(v \widetilde{a})\{\Psi \vdash \varphi\}$ where $\widetilde{a}$ are binding occurrences into $\Psi$ and $\varphi$. A solution of an atomic constraint is a pair $\left(\sigma, \Psi^{\prime}\right)$ where $\sigma$ is a substitution of terms for names such that $\widetilde{a} \# \sigma, \Psi^{\prime}$ and $\Psi \sigma \otimes \Psi^{\prime} \vdash \varphi \sigma$. We adopt the notation $(\sigma, \Psi) \models C$ to say that $(\sigma, \Psi)$ is a solution of $C$, and write $\operatorname{sol}(C)$ for $\{(\sigma, \Psi):(\sigma, \Psi) \models C\}$.

The transition constraints are the atomic constraints $C$ and conjunctions of atomic constraints $C \wedge C^{\prime}$, where the solutions are the intersection of the solutions for $C$ and $C^{\prime}$ and we let $(v \widetilde{a})\left(C \wedge C^{\prime}\right)$ mean $(v \widetilde{a}) C \wedge(v \widetilde{a}) C^{\prime}$. 


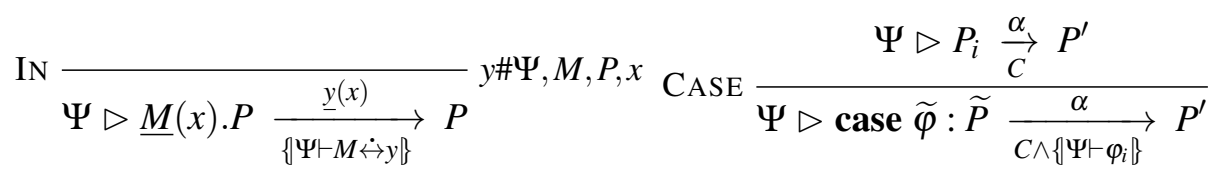

$$
\begin{aligned}
& \text { OUT } \underset{\Psi \triangleright \bar{M} N . P \underset{\{\Psi \vdash M \dot{4} y\}}{\longrightarrow} P}{\stackrel{\bar{y} N}{\Psi}} y \#, M, N, P
\end{aligned}
$$

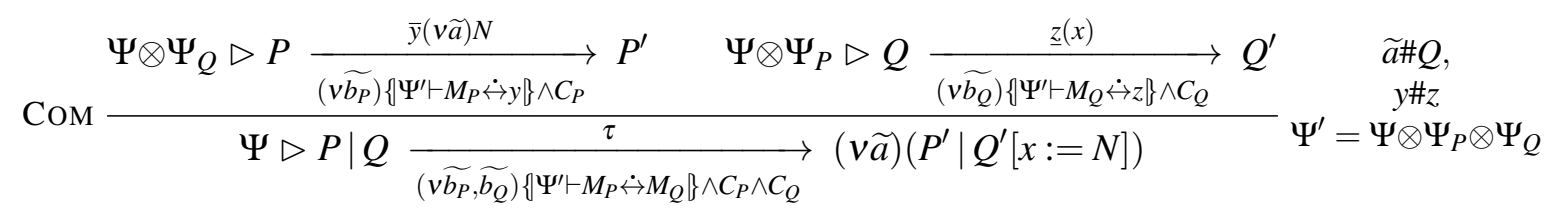

$$
\begin{aligned}
& \operatorname{PAR} \frac{\Psi \otimes \Psi_{Q} \triangleright P \underset{C}{\stackrel{\alpha}{\rightarrow}} P^{\prime}}{\Psi \triangleright P\left|Q \underset{\left(v \widetilde{b}_{Q}\right) C}{\stackrel{\alpha}{\longrightarrow}} P^{\prime}\right| Q} \alpha=\tau \vee \operatorname{subj}(\alpha) \# Q \quad \text { SCOPE } \frac{\Psi \triangleright P \underset{C}{\stackrel{\alpha}{\rightarrow} P^{\prime}}}{\Psi \triangleright(v a) P \frac{\alpha}{(v a) C}(v a) P^{\prime}} a \# \alpha, \Psi \\
& \operatorname{OPEN} \frac{\Psi \triangleright P \frac{\bar{y}(v \widetilde{a}) N}{C} P^{\prime}}{\Psi \triangleright(v a) P \frac{\bar{y}(v \widetilde{u} \cup a) N}{(v a) C} P^{\prime}} a \in \mathbb{\#} \widetilde{a}, \Psi, y \quad \text { REP } \frac{\Psi \triangleright P \mid ! P \underset{C}{\stackrel{\alpha}{\longrightarrow}} P^{\prime}}{\Psi \triangleright ! P \underset{C}{\stackrel{\alpha}{\longrightarrow}} P^{\prime}}
\end{aligned}
$$

Table 3: Transition rules for the symbolic semantics. Symmetric versions of COM and PAR are elided. In the rule COM we assume that $\mathscr{F}(P)=\left(v \widetilde{b}_{P}\right) \Psi_{P}$ and $\mathscr{F}(Q)=\left(v \widetilde{b}_{Q}\right) \Psi_{Q}$ where $\widetilde{b}_{P}$ is fresh for all of $\Psi, \widetilde{b}_{Q}, Q$ and $P$, and that $\widetilde{b}_{Q}$ is correspondingly fresh. We also assume that $y, z \# \Psi, \widetilde{b}_{P}, P, \widetilde{b}_{Q}, Q, N, \widetilde{b_{P}}, \widetilde{b_{Q}}, \widetilde{a}$. In the rule PAR we assume that $\mathscr{F}(Q)=\left(v \widetilde{b}_{Q}\right) \Psi_{Q}$ where $\widetilde{b}_{Q}$ is fresh for $\Psi, P$ and $\alpha$. In OPEN the expression $\tilde{a} \cup\{a\}$ means the sequence $\tilde{a}$ with $a$ inserted anywhere.

A transition constraint $C$ defines a set of solutions $\operatorname{sol}(C)$, namely those where the entailment becomes true by applying the substitution and adding the assertion. For example, the transition constraint $\{\mathbf{1} \vdash x=3\}$ has solutions $([x:=3], \mathbf{1})$ and (Id, $x=3)$, where Id is the identity substitution.

The structured operational symbolic semantics is defined in Table 3. First consider the OUT rule: $\Psi \triangleright \bar{M} N . P \underset{\{\Psi \vdash M \dot{\leftrightarrow} y\}}{\stackrel{\bar{y} N}{\longrightarrow}} P$. The symbolic subject $y$ must be chosen fresh and has a constraint associated with it: the transition can be taken in any solution that implies that the subject $M$ of the syntactic prefix is channel equivalent to $y$.

The rule COM is of particular interest. The intuition is that the symbolic action subjects are placeholders for the values $M_{P}$ and $M_{Q}$. In the conclusion the constraint is that these are channel equivalent, while $y$ and $z$ will not occur again.

We will often write $P \underset{C}{\stackrel{\alpha}{\rightarrow}} P^{\prime}$ for $\mathbf{1} \triangleright P \underset{C}{\stackrel{\alpha}{\rightarrow}} P^{\prime}$. 


\subsection{Symbolic bisimulation}

In order to define a symbolic bisimulation we need additional kinds of constraints. If a process $P$ does a bound output $\bar{y}(v \widetilde{a}) N$ that is matched by a bound output $\bar{y}(v \widetilde{a}) N^{\prime}$ from $Q$ we need constraints that keep track of the fact that $N$ and $N^{\prime}$ should be syntactically the same, and that $\widetilde{a}$ is sufficiently fresh.

Definition 14. The constraints include the transition constraints, $\{M=N\}$, and $\{a \# X\}$, where $X$ is any nominal data type. The solutions of the last two are all pairs $(\Psi, \sigma)$ such that $M \sigma=N \sigma$ and a\# $(X \sigma)$ respectively. We also include conjunction of constraints $C \wedge C^{\prime}$, where the set of solutions is the intersection of the solutions for $C$ and $C^{\prime}$.

Note that the assertion part of the solution is irrelevant for constraints of kind $\{M=N\}$ and $\{a \# X\}$, and that the substitution does not affect $a$ in $\{a \# X\}$. The constraint $\{M=N\}$ is used in the bisimulation for matching output objects, and $\{a \# X\}$ is used in the bisimulation for recording what an opened name must be fresh for. This corresponds to distinctions in open bisimulation for the pi-calculus [21]. We define true to be $\{M=M\}$, we write $\{a \# X, Y\}$ for $\{a \# X\} \wedge\{a \# Y\}$, and we extend the notation to sets of names, e.g. $\{\widetilde{a} \# X\}$.

Definition 15 (Constraint implication). A constraint $C$ implies another constraint $D$, written $C \Rightarrow D$, iff $\operatorname{sol}(C) \subseteq \operatorname{sol}(D)$. We write $C \Rightarrow \bigvee \widetilde{C}$ iff for each $(\sigma, \Psi) \in \operatorname{sol}(C)$ there exists a $C^{\prime} \in \widetilde{C}$ such that $(\sigma, \Psi) \in \operatorname{sol}\left(C^{\prime}\right)$.

Before we can give the definition of symbolic bisimulation we need to define a symbolic variant of the concrete static equivalence.

Definition 16 (Symbolic static equivalence). Two processes $P$ and $Q$ are statically equivalent for $C$, written $P \simeq_{C} Q$, if for each $(\sigma, \Psi) \in \operatorname{sol}(C)$ we have that $\Psi \otimes \mathscr{F}(P) \sigma \simeq \Psi \otimes \mathscr{F}(Q) \sigma$.

We now have everything we need to define symbolic bisimulation. This definition follows the definition in [17] closely.

Definition 17 ((Early) Symbolic bisimulation). A symbolic bisimulation $\mathscr{S}$ is a ternary relation between constraints and pairs of agents such that $\mathscr{S}(C, P, Q)$ implies all of

1. $P \simeq_{C} Q$, and

2. $\mathscr{S}(C, Q, P)$, and

3. If $P \underset{C_{P}}{\stackrel{\alpha}{\longrightarrow}} P^{\prime}, \operatorname{bn}(\alpha) \#\left(P, Q, C, C_{P}, \operatorname{subj}(\alpha)\right)$ and $\operatorname{subj}(\alpha) \#(P, Q, C)$ then there exists a set of constraints $\widehat{C}$ such that $C \wedge C_{P} \Rightarrow \bigvee \widehat{C}$

and for all $C^{\prime} \in \widehat{C}$ there exists $Q^{\prime}, \alpha^{\prime}$, and $C_{Q}$ such that

(a) $Q \underset{C_{Q}}{\stackrel{\alpha^{\prime}}{\rightarrow}} Q^{\prime}$, and

(b) $C^{\prime} \Rightarrow C_{Q}$, and

(c) if $\alpha=\bar{y}(v \widetilde{a}) N$ then $\alpha^{\prime}=\bar{y}(v \widetilde{a}) N^{\prime}, C^{\prime} \Rightarrow\left\{N=N^{\prime}\right\}$,

and $\left(C^{\prime} \wedge\{\widetilde{a} \# P, Q\}, P^{\prime}, Q^{\prime}\right) \in \mathscr{S}$

otherwise $\alpha=\alpha^{\prime}$ and $\left(C^{\prime}, P^{\prime}, Q^{\prime}\right) \in \mathscr{S}$

We write $P \sim_{s} Q$ if $($ true, $P, Q) \in \mathscr{S}$ for some symbolic bisimulation $\mathscr{S}$, and say that $P$ is symbolically bisimilar to $Q$.

The set $\widehat{C}$ allows a case analysis on the constraint solutions, as examplified in the next section. The output objects need to be equal in a solution to $C^{\prime}$. Since the solutions of $\left\{N=N^{\prime}\right\}$ only depend on the substitutions, this constraint corresponds to the fact that the objects must be identical in the concrete bisimulation. Note that $\operatorname{bn}(\alpha)$ may occur in $\widehat{C}$. Based on [9, 18], we conjecture that adding the requirement $\operatorname{bn}(\alpha) \# \widehat{C}$ would give late symbolic bisimulation. 


\section{Examples}

We now look at a few examples to illustrate the concrete and symbolic transitions and bisimulations. First consider a simple example from the pi-calculus. This can be expressed as a psi-calculus: let the only data terms be names, the only assertion be $\mathbf{1}$, the conditions be equality and inequality tests on names, and entailment defined by $\forall a . \mathbf{1} \vdash a=a, \forall a, b: a \neq b . \mathbf{1} \vdash a \neq b$ and $\forall a . \mathbf{1} \vdash a \dot{\leftrightarrow} a$. For a more thorough discussion, see [5]. In the following examples we drop a trailing .0. Consider the two agents $P_{1}$ and $Q_{1}$ :

$$
\begin{aligned}
P_{1}=a(x) \cdot P_{1}^{\prime} & \text { where } P_{1}^{\prime}=\bar{a} b \cdot \bar{a} b \\
Q_{1}=a(x) \cdot Q_{1}^{\prime} & \text { where } Q_{1}^{\prime}=(\text { case } x=b: \bar{a} b \cdot \bar{a} b \square x \neq b: \bar{a} b \cdot \bar{a} b)
\end{aligned}
$$

These are bisimilar. A concrete bisimulation between these agents is

$$
\left\{\left(\mathbf{1}, P_{1}, Q_{1}\right)\right\} \cup \bigcup_{n \in \mathscr{N}}\left\{\left(\mathbf{1}, P_{1}^{\prime}, Q_{1}^{\prime}[x:=n]\right\} \cup\{(\mathbf{1}, \bar{a} b, \bar{a} b)\}\right.
$$

The bisimulation needs to be infinite because of the infinite branching in the input. In contrast, a symbolic bisimulation only contains four triples:

$$
\left\{\left(\text { true }, P_{1}, Q_{1}\right),\left(\text { true }, P_{1}^{\prime}, Q_{1}^{\prime}\right),(\{\mathbf{1} \vdash x=b\}, \bar{a} b, \bar{a} b),(\{\mathbf{1} \vdash x \neq b\}, \bar{a} b, \bar{a} b)\right\}
$$

When checking the second triple (true, $\left.P_{1}^{\prime}, Q_{1}^{\prime}\right)$, the transition of $P_{1}^{\prime}$ is matched by a case analysis: $\widehat{C}$ in the definition of symbolic bisimulation (Def. 17) is $\{\{\mathbf{1} \vdash x=b\},\{\mathbf{1} \vdash x \neq b\}\}$, and a matching transition for $Q_{1}^{\prime}$ can be found for each of these cases, so the agents are bisimilar. In contrast, they are not equivalent in the incomplete symbolic bisimulations in [11] and [15].

Next we look at an example where we have tuples of channels and projection, e.g. the entailment relation gives us that $\mathbf{1} \vdash \operatorname{first}(M, N) \dot{\leftrightarrow} M$. Consider the agent

$$
R=\bar{M} N \cdot R^{\prime}
$$

Concretely this agent has infinitely many transitions even in an empty frame: $R \stackrel{\bar{M} N}{\longrightarrow} R^{\prime}$, and equivalent actions $\overline{\text { first }(M, K)} N$ for all $K$, and $\overline{\operatorname{first}(\operatorname{first}(M, L), K)} N$ for all $L$ and $K$, etc. Symbolically, however, it has only one transition: $R \underset{\{\mathbf{y} \vdash M \dot{y} N y\}}{\stackrel{\bar{y}}{\longrightarrow}} R^{\prime}$.

For another example, consider the two agents

$$
P_{2}=\bar{F} N \cdot P^{\prime} \quad Q_{2}=\mathbf{0}
$$

where $F$ is a term such that for no $\Psi, M$ does it hold that $\Psi \vdash F \dot{\leftrightarrow} M$, i.e., $F$ is not a channel. Then we have that $P_{2}$ and $Q_{2}$ are concretely bisimilar since neither one of them has a transition. But symbolically $P_{2}$ has the transition $P_{2} \underset{\{1 \vdash F \dot{\leftrightarrow} y\}}{\stackrel{\bar{y} N}{\longrightarrow}} P^{\prime}$, while $Q_{2}$ has no symbolic transition. Perhaps surprisingly they are still symbolically bisimilar: Def. 17 requires that we find a disjunction $\widehat{C}$ such that $C \wedge C_{P} \Rightarrow \bigvee \widehat{C}$, or in this case such that true $\wedge\{\mathbf{1} \vdash F \dot{\leftrightarrow} y\} \Rightarrow \bigvee \widehat{C}$. Since $F$ is not channel equivalent to anything, the left hand side has no solutions, which means that any set $\widehat{C}$ will do, and in particular the empty one. The condition "for all $C^{\prime} \in \widehat{C}$ " in the definition becomes trivially true, so $Q_{2}$ does not have to mimic the transition.

A final example shows the use of cryptographic primitives. Here the terms contains enc $(M, k)$ and $\operatorname{dec}(M, k)$, assertions are variable assignments, e.g. $x:=M$, the conditions are equality tests between 
terms, and the entailment relation is parametrised by an equation system which contains the equation $\operatorname{dec}(\operatorname{enc}(M, k), k)=M$. Consider

$$
\begin{aligned}
& P_{3}=(v a, k)((\mid x:=\operatorname{enc}(a, k)) \mid b(z) \cdot \bar{b} k \cdot(\text { case } z=a: \bar{c} d)) \\
& Q_{3}=(v a, k)((\mid x:=\operatorname{enc}(a, k)) \mid b(z) \cdot \bar{b} k)
\end{aligned}
$$

Here the environment can use $x$, the result of encrypting $a$ with $k$, but not the bound $a$ or $k$. Intuitively these agents are bisimilar since the key $k$ is not revealed until after the agents receive $z$, which therefore cannot be equal to $a$. The first symbolic transitions of the agents are

$$
\begin{aligned}
P_{3} \underset{(v a, k)\{\mathbf{1} \vdash b \dot{\leftrightarrow} y\}}{\stackrel{y(z)}{\longrightarrow}}(v a, k)((x:=\operatorname{enc}(a, k)) \mid \bar{b} k .(\text { case } z=a: \bar{c} d)) & =P_{3}^{\prime} \\
Q_{3} \underset{(v a, k)\{\mathbf{1} \vdash b \dot{\leftrightarrow} y\}}{\stackrel{y}{\longrightarrow}}(v a, k)((x:=\operatorname{enc}(a, k)) \mid \bar{b} k) & =Q_{3}^{\prime}
\end{aligned}
$$

and the second transitions are

$$
\begin{aligned}
& P_{3}^{\prime} \underset{(v a, k)\left\{\mathbf{1} \vdash b \dot{\leftrightarrow} \leftrightarrow y^{\prime}\right\}}{\longrightarrow}(v a)\left((x:=\operatorname{enc}(a, k) D \mid(\text { case } z=a: \bar{c} d))=P_{3}^{\prime \prime}\right. \\
& \left.Q_{3}^{\prime} \underset{(v a, k)\left\{1 \vdash b \dot{\leftrightarrow} y^{\prime}\right\}}{\stackrel{\overline{y^{\prime}}(v k) k}{\longrightarrow}}(v a)(\mid x:=\operatorname{enc}(a, k))\right) \quad=Q_{3}^{\prime \prime}
\end{aligned}
$$

A symbolic bisimulation, where we for simplicity ignore the constraints that arise for subjects, is

$$
\left\{\left(\text { true }, P_{3}, Q_{3}\right), \quad\left(\text { true }, P_{3}^{\prime}, Q_{3}^{\prime}\right), \quad\left(\left\{k \# P_{3}^{\prime}, Q_{3}^{\prime}\right\}, P_{3}^{\prime \prime}, Q_{3}^{\prime \prime}\right)\right\}
$$

Here the constraint $\left\{k \# P_{3}^{\prime}, Q_{3}^{\prime}\right\}$ will among other things imply that $k \# z$. The final transition of $P_{3}^{\prime \prime}$ has the constraint $(v a)\{\mathbf{1} \vdash z=a\}$, so we must find a disjunction $\widehat{C}$ such that $k \# P_{3}^{\prime}, Q_{3}^{\prime} \wedge(v a)\{\mathbf{1} \vdash z=a\} \Rightarrow \widehat{C}$. Since $a$ is bound, the only way to find a solution to the left hand side is to find a value for $z$ that evaluates to $a$. One candidate for a solution is $([z:=\operatorname{dec}(x, k)], \mathbf{1})$, but because of the constraint $k \# z$ this does not work. In fact, there is no solution to the left hand side because of the freshness constraint on $k$ and the fact that $a$ is bound. This means that, as in the previous example, any disjunction $\widehat{C}$ will do, and in particular the empty disjunction, and trivially $Q_{3}^{\prime \prime}$ does not have to mimic the transition.

In contrast, if we swap the order of the inputs and the outputs in $P_{3}$ and $Q_{3}$ and try to construct the bisimulation relation we will discover that we do not get the constraint $k \# z$. This means that $([z:=$ $\operatorname{dec}(x, k)], \mathbf{1})$ is a solution to $C \wedge C_{P}$ in the definition of bisimulation, and that $Q_{3}^{\prime \prime}$ must mimic the transition from $P_{3}^{\prime \prime}$. In this case the agents are not bisimilar.

\section{Results}

We now turn to showing that the concrete and symbolic equivalences coincide.

We define substitution on symbolic actions by $\tau \sigma=\tau,(y(x)) \sigma=y \sigma(x \sigma)$, and $(\bar{y}(v \widetilde{a}) N) \sigma=$ $\overline{y \sigma}(v \widetilde{a}) N \sigma$, where $x, \widetilde{a} \# \sigma$. We define the substitution $\sigma \cdot[y:=M]$ for $y \# \bar{\sigma}$ by $(\sigma \cdot[y:=M])(x)=M$ if $x=y$, and $\sigma(x)$ otherwise.

The following two lemmas show the operational correspondence between the symbolic semantics and the concrete semantics: given a symbolic transition where the transition constraint has a solution, there is always a corresponding concrete transition (Lemma 18) and vice versa (Lemma 19).

Lemma 18 (Correspondence symbolic-concrete). 
1. If $P \stackrel{\underline{y}(x)}{\longrightarrow} P^{\prime}$ then for all $(\sigma, \Psi) \in \operatorname{sol}(C)$ s.t. $x \# \sigma$ we have that $\Psi \triangleright P \sigma \stackrel{(y(x)) \sigma}{\longrightarrow} P^{\prime} \sigma$.

2. If $P \stackrel{\bar{y}(v \widetilde{a}) N}{\longrightarrow} P^{\prime}$ then for all $(\sigma, \Psi) \in \operatorname{sol}(C)$ s.t. $\widetilde{a} \# \sigma$ we have that $\Psi \triangleright P \sigma \stackrel{(\bar{y}(v \widetilde{a}) N) \sigma}{\longrightarrow} P^{\prime} \sigma$.

3. If $P \underset{C}{\stackrel{\tau}{\longrightarrow}} P^{\prime}$ then for all $(\sigma, \Psi) \in \operatorname{sol}(C)$ we have that $\Psi \triangleright P \sigma \stackrel{\tau}{\longrightarrow} P^{\prime} \sigma$.

Lemma 19 (Correspondence concrete-symbolic).

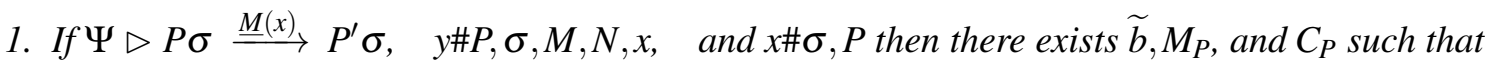
$P \underset{\left.(v \widetilde{b})\left\{\Psi_{P} \vdash M_{P} \dot{\leftrightarrow} y\right\}\right\} \wedge C_{P}}{\longrightarrow} P^{\prime}$ and $(\sigma \cdot[y:=M], \Psi) \in \operatorname{sol}\left((v \widetilde{b})\left\{\left\{\Psi_{P} \vdash M_{P} \dot{\leftrightarrow} y\right\} \wedge C_{P}\right)\right.$.

2. If $\Psi \triangleright P \sigma \stackrel{\bar{M}(v \widetilde{a}) N \sigma}{\longrightarrow} P^{\prime} \sigma, \quad y \# P, \sigma, M, \widetilde{a}, \quad$ and $\widetilde{a} \# \sigma, P$ then there exists $\widetilde{b}, M_{P}, C_{P}$ such that $P \underset{(v \widetilde{b})\left\{\Psi_{P} \vdash M_{P} \dot{\leftrightarrow} \rightarrow y\right\} \wedge C_{P}}{\longrightarrow} P^{\prime}$ and $(\sigma \cdot[y:=M], \Psi) \in \operatorname{sol}\left((v \widetilde{b})\left\{\Psi_{P} \vdash M_{P} \dot{\leftrightarrow} y\right\} \wedge C_{P}\right)$.

3. If $\Psi \triangleright P \sigma \stackrel{\tau}{\longrightarrow} P^{\prime} \sigma$ then there exists $C$ such that $P \underset{C}{\stackrel{\tau}{\rightarrow}} P^{\prime}$ and $(\sigma, \Psi) \in \operatorname{sol}(C)$.

We assume in 1 and 2 that $\mathscr{F}(P)=\left\langle\widetilde{b}_{P}, \Psi_{P}\right\rangle$ and $\widetilde{b}_{P}, \widetilde{b} \# y, \Psi, \sigma, P$.

The proofs are by induction over the transition derivation (one case for each rule).

Theorem 20 (Soundness). Assume $\mathscr{S}$ is a symbolic bisimulation and let $\mathscr{R}=\{(\Psi, P \sigma, Q \sigma): \exists C .(\sigma, \Psi) \models C$ and $(C, P, Q) \in \mathscr{S}\}$. Then $\mathscr{R}$ is a concrete bisimulation.

The proof idea to show that $\mathscr{R}$ is a concrete bisimulation is to assume $(\Psi, P \sigma, Q \sigma) \in \mathscr{R}$ and that $P \sigma$ has a transition in environment $\Psi$. We use Lemma 19 to find a symbolic transition from $P$, then the fact that $\mathscr{S}$ is a symbolic bisimulation to find a simulating symbolic transition from $Q$, and finally Lemma 18 to find the required concrete transitions from $Q \sigma$.

Similarly to [17] we need an extra assumption about the expressiveness of constraints: for all $\mathscr{R}, P, Q$ such that $\mathscr{R}$ is a concrete bisimulation there exists a constraint $C$ such that $(\Psi, \sigma) \models C \Longleftrightarrow$ $(\Psi, P \sigma, Q \sigma) \in \mathscr{R}$. In order to determine symbolic bisimilarity in an efficient way we need to compute this constraint, which is easy for the pi-calculus [9, 18, 19] and harder (but in many practical cases possible) for cryptographic signatures [10]. These results suggest that our constraints are sufficiently expressive, but for other instances of psi-calculi we may have to extend the constraint language. We leave this as an area of further research.

Theorem 21 (Completeness). Assume that $\mathscr{R}$ is a concrete bisimulation and let $\mathscr{S}=\{(C, P, Q):(\sigma, \Psi) \models C$ implies $(\Psi, P \sigma, Q \sigma) \in \mathscr{R}\}$. Then $\mathscr{S}$ is a symbolic bisimulation.

The proof idea is the converse of the proof for Theorem 20. The expressiveness assumption of constraints mentioned above is needed in order to construct the disjunction of constraints in the symbolic bisimulation. From these two theorems we get:

Corollary 22 (Full abstraction). $P \sim Q$ if and only if $P \sim_{s} Q$.

\section{Conclusion and Future Work}

We have defined a symbolic operational semantics for psi-calculi and a symbolic bisimulation which is fully abstract wrt the original semantics. While the developments in [5] give meta-theory for a wide 
range of calculi of mobile processes with nominal data and logic, the work presented in this paper gives a solid foundation for automated tools for the analysis of such calculi.

As mentioned in the introduction, the purity of the original semantics of psi-calculi has made the symbolic semantics easier to develop. There are no structural equivalence rules (which are a complication in applied pi-calculus), the scope opening rule is because of this straight-forward which makes knowledge representation simpler than in spi-calculi, and the bisimulation less complex. Nevertheless, the technical challenges have not been absent: the precise design of the constraints and their solution has been delicate. Since assertions may occur under a prefix, the environment can change after a transition. Keeping the assertion $\Psi$ in the transition constraints (on the form $(v \widetilde{a})\{\Psi \vdash \varphi\}$ ) essentially keeps a snapshot of the environment that gives rise to the transition. An alternative would be to use time stamps to keep track of which environment made which condition true, but that approach seems more difficult.

Our symbolic bisimulation is a strong equivalence which does not abstract the internal $\tau$ transitions. This is less useful for verification than a weak observational equivalence, but still a significant step towards mechanized verification. We are currently developing a weak bisimulation for psi-calculi, and are studying the correspondence to a barbed bisimulation congruence. Preliminary results indicate that lifting the symbolic bisimulation presented here to weak bisimulation will be unproblematic.

The original psi-calculi admit pattern matching in inputs. In a symbolic semantics this would lead to complications in the COM-rule, which should introduce a substitution for the names bound in the pattern. This means introducing more fresh names and constraints, and it is not clear that the convenience of pattern matching outweighs such an awkward semantic rule. We leave this as an area for further study.

For future work, we need to develop an algorithm for deciding symbolic bisimulation and implement it in a tool. A natural basis for this would be the algorithm given in [17]. Furthermore, the termination of the algorithm will depend on the properties of the parameters of the particular psi-calculus: it is easy to construct a psi-calculus where the entailment relation or static equivalence is not decidable, but in many practical cases it will be [10, 4]. We intend to use constraint solvers developed for specific application domains (e.g. security) in a future generic tool. We will also produce mechanized proofs of the adequacy of the symbolic semantics, using the Isabelle theorem prover.

When typing schemes have been developed for psi-calculi, a natural progression would be to take advantage of those also in the symbolic semantics, to further constrain the possible values and thus the size of state spaces.

\section{References}

[1] Martín Abadi \& Bruno Blanchet (2005): Analyzing Security Protocols with Secrecy Types and Logic Programs. Journal of the ACM 52(1), pp. 102-146.

[2] Martín Abadi \& Cédric Fournet (2001): Mobile Values, New Names, and Secure Communication. In: Proceedings of POPL '01. ACM, pp. 104-115.

[3] Martín Abadi \& Andrew D. Gordon (1999): A Calculus for Cryptographic Protocols: The Spi Calculus. Journal of Information and Computation 148(1), pp. 1-70.

[4] Mathieu Baudet (2007): Sécurité des protocoles cryptographiques: aspects logiques et calculatoires. Ph.D. thesis, École Normale Supérieure de Cachan.

[5] Jesper Bengtson, Magnus Johansson, Joachim Parrow \& Björn Victor (2009): Psi-calculi: Mobile processes, nominal data, and logic. In: Proceedings of LICS 2009. IEEE, Computer Society Press, pp. 39-48.

[6] Jesper Bengtson \& Joachim Parrow (2009): Psi-calculi in Isabelle. In: Stefan Berghofer, Tobias Nipkow, Christian Urban \& Makarius Wenzel, editors: Proc. of TPHOLs 2009, LNCS 5674. Springer, pp. 99-114. 
[7] Bruno Blanchet (2001): An Efficient Cryptographic Protocol Verifier Based on Prolog Rules. CSFW'01: Proceedings of the 14th IEEE Computer Security Foundations Workshop , p. 82.

[8] Bruno Blanchet, Martín Abadi \& Cédric Fournet (2005): Automated Verification of Selected Equivalences for Security Protocols. In: 20th IEEE Symposium on Logic in Computer Science (LICS 2005). IEEE, Computer Society Press, pp. 331-340.

[9] Michele Boreale \& Rocco De Nicola (1996): A Symbolic Semantics for the $\pi$-Calculus. Journal of Information and Computation 126(1), pp. 34-52. Available as Report SI 94 RR 04, Università "La Sapienza" di Roma; an extended abstract appeared in Proceedings of CONCUR '94, pages 299-314, LNCS 836.

[10] Johannes Borgström (2008): Equivalences and Calculi for Formal Verifiation of Cryptographic Protocols. Ph.D. thesis, EPFL, Lausanne.

[11] Johannes Borgström, Sébastien Briais \& Uwe Nestmann (2004): Symbolic Bisimulation in the Spi Calculus. In: Proceedings of CONCUR 2004, LNCS 3170. Springer, pp. 161-176.

[12] Johannes Borgström \& Uwe Nestmann (2002): On Bisimulations for the Spi Calculus. In: Hélène Kirchner \& Christophe Ringeissen, editors: Proceedings of AMAST 2002, LNCS 2422. Springer, pp. 287-303.

[13] Sébastien Briais (2008): Theory and Tool Support for the Formal Verification of Cryptographic Protocols. Ph.D. thesis, EPFL, Lausanne.

[14] Maria Grazia Buscemi \& Ugo Montanari (2008): Open Bisimulation for the Concurrent Constraint Picalculus. In: Sophia Drossopoulou, editor: Proceedings of ESOP 2008, LNCS 4960. Springer, pp. 254-268.

[15] Stéphanie Delaune, Steve Kremer \& Mark Ryan (2007): Symbolic Bisimulation for the Applied Pi Calculus. In: Vikraman Arvind \& Sanjiva Prasad, editors: Proc. of FSTTCS 2007, Lecture Notes in Computer Science 4855. Springer, pp. 133-145. Available at http://dx.doi.org/10.1007/978-3-540-77050-3_11.

[16] Murdoch Gabbay \& Andrew Pitts (2001): A New Approach to Abstract Syntax with Variable Binding. Formal Aspects of Computing 13, pp. 341-363.

[17] Matthew Hennessy \& Huimin Lin (1995): Symbolic Bisimulations. Theoretical Computer Science 138(2), pp. 353-389. Earlier version published as Technical Report 1/92, School of Cognitive and Computing Sciences, University of Sussex, UK.

[18] Huimin Lin (1996): Symbolic Transition Graph with Assignment. In: Ugo Montanari \& Vladimiro Sassone, editors: Proceedings of CONCUR '96, LNCS 1119. Springer, pp. 50-65.

[19] Huimin Lin (2000): Computing Bisimulations for Finite-Control pi-Calculus. Journal of Computer Science and Technology 15(1), pp. 1-9.

[20] A. M. Pitts (2003): Nominal Logic, A First Order Theory of Names and Binding. Information and Computation 186, pp. 165-193.

[21] Davide Sangiorgi (1996): A Theory of Bisimulation for the $\pi$-calculus. Acta Informatica 33, pp. 69-97. Earlier version published as Report ECS-LFCS-93-270, University of Edinburgh. An extended abstract appeared in the Proceedings of CONCUR '93, LNCS 715.

[22] Björn Victor \& Faron Moller (1994): The Mobility Workbench - A Tool for the $\pi$-Calculus. In: David Dill, editor: Proceedings of CAV '94, LNCS 818. Springer, pp. 428-440. 